Original Article

\title{
A RANDOMIZED CONTROLLED TRIAL OF CURCUMIN AND DICLOFENAC COMBINATION IN KNEE OSTEOARTHRITIS
}

\author{
DHANESHWAR SHEP1 ${ }^{\text {, CHITRA KHANWELKAR¹, PRAKASHCHANDRA GADE² }}{ }^{2}$ SATYANAND KARAD ${ }^{3}$ \\ ${ }^{1}$ Department of Pharmacology, Krishna Institute of Medical Sciences, Karad, Maharashtra, India, ${ }^{2}$ Department of Pharmacology, Dr. \\ Vithalrao Vikhe Patil Foundation's Medical College and Hospital, Ahmednagar, Maharashtra, India, ${ }^{3}$ Department of Orthopedics, City Care \\ Accident Hospital, Parli Vaijnath, Beed, Maharashtra, India \\ Email: dhaneshshep@gmail.com
}

Received: 13 Aug 2019, Revised and Accepted: 18 Oct 2019

\section{ABSTRACT}

Objective: The objective of the study was to evaluate pain relief and safety of the combination of curcumin and diclofenac versus diclofenac alone in the treatment of knee osteoarthritis (OA).

Methods: 140 patients of knee OA meeting inclusion criteria were randomized to receive either curcumin $500 \mathrm{mg}$ with diclofenac $50 \mathrm{mg}$ twice daily or diclofenac $50 \mathrm{mg}$ tablet alone twice daily for $28 \mathrm{~d}$. Patients were assessed at baseline, Day 14 and Day 28. Primary efficacy measure was severity of pain (Visual Analogue Scale) at day 14 and day 28. Safety after treatment was evaluated by recording side effects and laboratory investigations.

Results: Patients receiving curcumin plus diclofenac showed significantly superior improvement in severity of pain at each study visit ( $<<0.001$ ) when compared to diclofenac. Adverse effects were significantly less in curcumin plus diclofenac group $(p<0.001)$.

Conclusion: Combination of curcumin and diclofenac showed a significant improvement in pain on the basis of VAS when compared to diclofenac which may be due to synergistic effect between curcumin and diclofenac

Keywords: Knee osteoarthritis, Curcumin, Diclofenac, Pain

(c) 2019 The Authors. Published by Innovare Academic Sciences Pvt Ltd. This is an open access article under the CC BY license (http://creativecommons.org/licenses/by/4.0/) DOI: http://dx.doi.org/10.22159/ijcpr.2019v11i6.36355

\section{INTRODUCTION}

Osteoarthritis is characterized by the breakdown of cartilage, joint lining, ligaments, and underlying bone [1-3]. It typically involves an entire joint, with the most commonly affected joints being the knees, hips, hands, and spine. Common manifestations of osteoarthritis are pain and stiffness. There are variety of risk factors for osteoarthritis, including obesity, high-impact sports, and bone deformities. The prevalence of osteoarthritis increases with age.

Osteoarthritis $(\mathrm{OA})$ is the most common form of arthritis and one of the leading causes of disability. This degenerative and progressive joint disease affects around 250 million people worldwide [4] and more than 27 million people in the United States $[5,6]$. The estimated prevalence of knee osteoarthritis in populations above the age of 65 is $30 \%$.

The knee is the largest synovial joint in humans, it is composed by osseous structures, cartilage, ligaments and a synovial membrane. The latter is in charge of the production of the synovial fluid, which provides lubrication and nutrients to the avascular cartilage [7]. Unfortunately, given the high use and stress of this joint, it is a frequent site for painful conditions, including OA [8]. In OA of knee, pain is the key symptom that drives individuals to seek medical attention and contributes to functional limitations and reduced quality of life [9-12].

The vast majority of $\mathrm{OA}$ patients are elderly and most of them will have multiple comorbidities. Hence, special attention should be paid to the possible interactions and adverse effects that systemic medications can induce in this population. Cyclooxygenase inhibitors (NSAIDs) have been the most commonly used medications. But given the gastrointestinal, renal, cardiac, and hematological adverse effects of these medications, their long-term use is limited.

Dietary supplements, including herbal products, have also been examined for the treatment of osteoarthritis. Several dietary supplements (eg, glucosamine, glucosamine with chondroitin) have demonstrated efficacy compared to placebo and active controls. One additional product that has been evaluated and used for the treatment of osteoarthritis is curcumin [13]. Curcumin is an active constituent that is derived from the rhizome of turmeric (Curcuma longa).

It is a yellow substance commonly used as a food coloring and as an ingredient in curry. Curcuma has a long history of being used in complementary and alternative medicine, and is commonly taken for a variety for health conditions such as arthritis, gastrointestinal complaints, respiratory infections, and even cancer. There is some evidence that shows curcuma has anti-inflammatory, antithrombotic, antioxidant, and antimicrobial activities. The exact mechanism of action associated with curcumin is not fully understood. The anti-inflammatory effects of curcumin are believed to be a result of inhibiting proinflammatory signals such as prostaglandins, leukotrienes, and cyclooxygenase-2. A clinical trial conducted by Shep et al., has shown that the efficacy of curcumin $(1500 \mathrm{mg} /$ day $)$ is similar to that of diclofenac (100 mg/day) for the treatment of knee osteoarthritis [14].

Combination of drugs with a different mode of actions may be more productive and less toxic than monotherapies. NSAIDs are often coadministered with proton-pump inhibitors or $\mathrm{H} 2$ blockers to reduce NSAID induced gastrointestinal adverse events. Evidence on the clinical effectiveness of curcumin and diclofenac in patients with knee osteoarthritis is lacking.

The objective of this study was to evaluate pain relief and safety of combination of curcumin and diclofenac as compare to diclofenac alone in a patient with $\mathrm{OA}$ of the knee by using Visual Analogue Scale.

\section{MATERIALS AND METHODS}

\section{Ethics and participant confidentiality}

This study was conducted in accordance with the Declaration of Helsinki, the ICH-GCP E6 (R1, R2), and ICMR-National Ethical Guidelines for Biomedical and Health Research 2006. Ethics committee approval was obtained from the Krishna Institute of Medical Sciences, Karad, Maharashtra, India, before initiating the study. Prior to any study-related 
screening procedures, written informed consent was obtained by the principal investigator from each patient before enrolment in the study. The study was registered with the ISRCTN registry (ISRCTN10074826). Each participant was identified only by the participant study number, and all documents in the study were identified by using the initials and participant study number.

\section{Trial design and participants}

In this randomized, open-label, active-controlled parallel group study, the patients of either gender (aged 38-65 y) suffering from symptomatic $\mathrm{OA}$ of knee for at least 3 mo with no joint deformities and requiring treatment with anti-inflammatory drugs were screened for eligibility after taking written informed consent. Patient meeting the American College of Rheumatology (ACR) criteria for OA of the knee (confirmed by X-ray) and having moderate pain (Visual analogue scale score 4 or greater) in disease joint were included in the study. Patients taking analgesics were given a washout period of at least 3-7 d before starting the study drug.

Patient who received corticosteroid injection of any drug within last $4 \mathrm{w}$; had a history of active peptic ulcer, gastric ulceration, stomach pain or GI bleeding or bleeding disorders; had secondary osteoarthritis due to syphilis, metabolic bone disorder, acute trauma; patients who required prescription anticoagulants, steroids or concurrent pain-relieving medication such as tranquilizers, hypnotics, excessive alcohol or any other drug affecting the evaluation of analgesic action, or patient having known hypersensitivity to diclofenac sodium and turmeric were excluded from the study. The patients with a medical history of significant impairment of hepatic or renal functions, cardiac insufficiency, and bronchitis were also excluded. Pregnant and lactating women and women of childbearing age not practicing or not willing to use contraceptives were not included.

\section{Interventions and dosage}

Patients fulfilling the eligibility criteria were enrolled and randomized to receive either diclofenac $50 \mathrm{mg}$ tablet (manufactured by Lupin Pharmaceuticals, Mumbai) twice daily or curcumin $500 \mathrm{mg}$ plus diclofenac $50 \mathrm{mg}$ (individual capsule and tablet were administered simultaneously, twice daily for $28 \mathrm{~d}(4 \mathrm{w})$. Curcumin $500 \mathrm{mg}$ capsule was provided by Arjuna Natural Pvt. Ltd, India. Paracetamol $500 \mathrm{mg}$ Tab (manufactured by GlaxoSmithKline Pharmaceuticals Limited) and Ranitidine $150 \mathrm{mg}$ Tab (manufactured by J B Chemicals and Pharma Ltd, Mumbai, India) were provided to Patients as rescue medication as and when required.

Simple randomization sequence distinct was generated by the computerized method before start of the study with equal distribution (allocation ratio 1:1) of the patients in both the treatment groups.

\begin{abstract}
Assessments
Patients were evaluated for efficacy at week 2 (Day 14) and week 4 (Day 28) after the start of the study treatment from baseline. Primary endpoints were improvement in the pain intensity on the basis of visual analogue scale (VAS). Secondary endpoints were to determine patient's global assessment for overall symptom relief and physician's global evaluation of treatment. Requirement of rescue medication throughout the study period was recorded in case report form. Side effects reported/observed during the study period were recorded in case report form at each study visit.
\end{abstract}

\section{Statistical analysis}

Sample size calculation was performed using software PS Power and Sample Size Calculations (version no.3). Based on a power of $80 \%$ and a Type I error rate of alpha $=0.05$ (2-tailed), a sample size of 65 participants per group was required to detect an estimated difference of 1.24 in the mean pain scores between the treatment arms with standard deviation (SD) of 2.5. Assuming the dropout rate of $5 \%$, a total sample size of 69 participants per treatment group was considered sufficient in this study.

All statistical analyses were performed on an intention-to-treat basis, with last observation carried forward method. Unpaired t test or Mann Whitney test was used to compare the data between groups and paired t-test or Wilcoxon signed-rank test was used for within-group analysis of the continuous data based on the distribution of data and Chi-square test or Fishers exact test was used to compare the categorical data of study groups. For comparing VAS score between the groups independent t-test was used as the scores were not on one of the extremes. P-value of less than 0.05 was considered as statistically significant. All statistical analyses were performed using software, SPSS version 24 .

\section{RESULTS}

\section{Patient disposition and characteristics}

Total 150 patients were enrolled in the study. A total of 140 patients (curcumin plus diclofenac: 71; diclofenac: 69) completed the study and were subjected to statistical analysis (fig. 1). Both treatment groups were comparable in terms of demographic characteristics i.e. age, weight, height and gender. Clinical assessment of pain on VAS at the start of the trial (baseline) was similar between both treatment groups. Overall, demography and baseline characteristics between both the treatment groups were similar before start of study treatment (table 1).

Table 1: Demography and baseline characteristics in patients with $\mathrm{OA}$ of knee

\begin{tabular}{lll}
\hline Patient characteristic & Curcumin+Diclofenac (N=71) & Diclofenac (N=69) \\
\hline Age (y) & $52.55 \pm 4.46$ & $52.14 \pm 3.76$ \\
Gender (M/F) & $\mathrm{M}=50 ; \mathrm{F}=21$ & $\mathrm{M}=48 ; \mathrm{F}=21$ \\
Weight (Kg) & $62.32 \pm 5.69$ & $63.51 \pm 5.19$ \\
Duration of osteoarthritis of knee (months) & $7.41 \pm 2.92$ & $7.45 \pm 3.15$ \\
Baseline pain intensity on Visual analogue scale (VAS) & $7.90 \pm 0.64$ & $7.81 \pm 0.73$ \\
\hline
\end{tabular}

Note: Values are expressed in mean \pm standard deviation except for gender variable (presented as number of patients in each category). Visual analogue scale is from 0 to 10 , where 0 indicates "No pain" and 10 indicates "Worst possible pain"

Table 2: Comparison of pain as determined by VAS in patients with OA of knee

\begin{tabular}{llll}
\hline Visit & Curcumin+Diclofenac (N=71) & Diclofenac (N=69) & p value \\
\hline Baseline & $7.90 \pm 0.64$ & $7.81 \pm 0.73$ & $.43^{\mathrm{t}}$ \\
Day 14 & $3.73 \pm 0.83$ & $4.58 \pm 0.60$ & $<.001^{\mathrm{t}}$ \\
Day 28 & $1.38 \pm 0.74$ & $2.20 \pm 0.61$ & $<.001^{\mathrm{t}}$ \\
Change at Day 14 & $4.17 \pm 0.88$ & $3.23 \pm 0.91$ & $<.001^{\mathrm{t}}$ \\
Change at Day 28 & $6.52 \pm 0.89$ & $5.61 \pm 0.88$ & $<.001^{\mathrm{t}}$ \\
p value (within group) & $<.001^{\mathrm{wc}}$ & $<.001^{\mathrm{wc}}$ & \\
VAS Reduction \% 5 50 & $\mathrm{N}=0$ & $\mathrm{~N}=2$ & $\mathrm{P}=.24^{\mathrm{f}}$ \\
VAS Reduction $\%>50$ & $\mathrm{~N}=71$ & $\mathrm{~N}=67$ & \\
\hline
\end{tabular}

Note: Values are expressed in mean \pm standard deviation. Data were analyzed by Wilcoxon signed-rank test (wc), Independent T-test ( $t$ ), Fisher Exact test (f). $\mathrm{N}=$ number of patients in each group. $\mathrm{p}<.05$ considered as a statistical significant difference, $\mathrm{OA}=$ osteoarthritis, VAS $=$ Visual analogue scale $(0$ to 10 where 0 indicates "No pain" and 10 indicate "Worst possible pain"). Change in mean score at Day 14 and 28 is calculated from baseline VAS score. 


\section{Efficacy results}

Both treatment groups showed a consistent decline in pain intensity on VAS at each study visit from baseline. However, patients treated with curcumin plus diclofenac experienced a significantly greater reduction in pain intensity compared to those treated with diclofenac alone at each study visit on day 14 and $28(\mathrm{p}<0.001) .71$ patients in curcumin plus diclofenac group and 67 patients in diclofenac group had more than $50 \%$ improvement in VAS score and the difference when compared were not statistically significant $(p=0.24)$ (table 2).

Global assessments of treatment by patient and physician based on overall pain relief and safety was favourable towards curcumin plus diclofenac compared to diclofenac alone. Greater proportion of patients rated curcumin plus diclofenac as excellent than diclofenac.

\section{Safety variables}

Overall, $13 \%$ of patients receiving curcumin plus diclofenac and $38 \%$ of patients receiving diclofenac reported adverse effects, the difference was statistically significant $(\mathrm{p}<0.001)$. All reported adverse effects were mild and transient. The most common adverse effects were nausea, diarrhea, abdominal pain and acidity; however, the incidence of each side effect was significantly lesser in curcumin plus diclofenac compared to the diclofenac group.

\section{DISCUSSION}

Currently, available medication regimens for the treatment of knee OA include paracetamol and NSAIDs, including COX-2 inhibitors. However, long-term use of NSAIDs has been found to be associated with enhanced risk for gastrointestinal bleeding and renal insufficiency. There is need of effective and safer alternative treatments for patients with $\mathrm{OA}$ of knee.

This study demonstrated that curcumin plus diclofenac administered twice daily for $28 \mathrm{~d}$ in patients with OA of knee was superior to diclofenac administered twice daily for primary endpoint (Pain intensity score on VAS) at each evaluation visits, indicating better pain relief with a combination of curcumin and diclofenac.

The favorable pain relief of combination therapy was observed due to analgesic/anti-inflammatory properties of curcumin that has been attributed to its ability to inhibit COX-2, which results in the suppression of prostaglandin synthesis. Further, curcumin has been shown to suppress pro-inflammatory cytokines and mediators of their release such as tumour necrosis factor, interleukin-1, IL-8 and nitric oxides synthase [15].

Several studies have demonstrated that curcumin functions as an antioxidant, anti-inflammatory, and anti-atherosclerotic; inhibits scarring, cataract, and gallstone formation; promotes wound healing and muscle regeneration; prevents liver injury and kidney toxicity; and exerts medicinal benefits against psoriasis, diabetes, multiple sclerosis, Alzheimer's, HIV disease, septic shock, cardiovascular disease, lung fibrosis, arthritis, and inflammatory bowel disease [16-18].

In the present study, it is found that less number of patients required additional rescue analgesics while receiving a combination of curcumin and diclofenac compared to diclofenac monotherapy depicting more stable pain control with combination therapy. Further, significantly less number of patients in curcumin plus diclofenac group reported side effects compared with diclofenac monotherapy. This favorable pain relief and safety profile in curcumin plus diclofenac may be due to the concurrent use of drugs with different mechanisms of action.

Based on overall pain relief and safety results, the patient's and physician's global evaluation of treatments favored combination therapy of curcumin and diclofenac than diclofenac monotherapy, which reflects the better acceptability of combination therapy of NSAIDs and curcumin among patients with OA of knee.

This study suggest that the combination therapy of curcumin and diclofenac is more effective in pain relief as compare to diclofenac alone in patients with OA of knee. Significant reduction was observed in GI side effects in patients who received diclofenac plus curcumin as compare to those who received only diclofenac.

\section{CONCLUSION}

Combination of curcumin and diclofenac showed a significant improvement in pain on the basis of VAS when compared to diclofenac which may be due to synergistic effect between curcumin and diclofenac.

\section{ACKNOWLEDGMENT}

All authors would like to thank the subjects for their participation in this study, Dr. Rakesh Ojha for performing statistical analysis and Arjuna Natural Pvt. Ltd, India, for providing curcumin capsules as study samples.

\section{FUNDING}

Nil

\section{AUTHORS CONTRIBUTIONS}

All the authors have contributed equally.

\section{CONFLICTS OF INTERESTS}

The authors declare that they have no conflict of interest

\section{REFERENCES}

1. Buys LM, Elliott M. Osteoarthritis. In: DiPiro JT, Talbert RL, Yee GC, Matzke GR, Wells BG, Posey L. eds. Pharmacotherapy: a pathophysiologic approach. 9th ed. New York, NY: McGrawHill; 2014. p. 1437-58.

2. Altman R, Asch E, Bloch G. Development of criteria for the classification and reporting of osteoarthritis: classification of osteoarthritis of the knee. Arthritis Rheum 1986; 29:1039-49.

3. Hochberg MC, Altman RD, April KT. American college of rheumatology 2012 recommendations for the use of nonpharmacologic and pharmacologic therapies in osteoarthritis of the hand, hip, and knee. Arthritis Care Res 2012;64:465-74.

4. Bannuru RR, Schmid CH, Kent DM, Vaysbrot EE, Wong JB, Mcalindon TE. Comparative effectiveness of pharmacologic interventions for knee osteoarthritis: a systematic review and network meta-analysis. Ann Int Med 2015;162:46-54.

5. Nelson AE, Allen KD, Golightly YM, Goode AP, Jordan JM. A systematic review of recommendations and guidelines for the management of osteoarthritis: the chronic osteoarthritis management initiative of the U. S. bone and joint initiative. Semin Arthritis Rheum 2014;43:701-12.

6. Da Costa BR, Hari R, Juni P. Intra-articular corticosteroids for osteoarthritis of the knee. JAMA 2016;316:2671-2.

7. Sharma V, Anuvat K, John L, Davis M. Scientific american pain management-arthritis of the knee. Decker: Pain-related disease states; 2017.

8. Richebe P, Capdevila X, Rivat C. Persistent postsurgical pain: pathophysiology and preventative pharmacologic considerations. Anesthesiology 2018;129:590-607.

9. Hadler NM. Knee pain is the malady-not osteoarthritis. Ann Intern Med 1992;116:598-9.

10. Ayis S, Dieppe P. The natural history of disability and its determinants in adults with lower limb musculoskeletal pain. J Rheumatol 2009;36:583-91.

11. Dominick KL, Ahern FM, Gold CH, Heller DA. Health-related quality of life and health service use among older adults with osteoarthritis. Arthritis Rheum 2004;51:326-31.

12. McAlindon TE, Cooper C, Kirwan JR, Dieppe PA. Determinants of disability in osteoarthritis of the knee.Ann Rheum Dis 1993;52:258-62.

13. Prasad S, Tyagi AK, Aggarwal BB. Recent developments in delivery, bioavailability, absorption and metabolism of curcumin: the golden pigment from golden spice. Cancer Res Treat 2014;46:2-18.

14. Dhaneshwar Shep, Chitra Khanwelkar, Prakashchandra Gade, Satyanand Karad. Safety and efficacy of curcumin versus diclofenac in knee osteoarthritis: a randomized open-label parallel-arm study. Trials 2019;20:214.

15. Henrotin Y, Priem F, Mobasheri Ali. Curcumin: a new paradigm and therapeutic opportunity for the treatment of osteoarthritis: 
curcumin for osteoarthritis management. Springerplus 2013;2:56.

16. Aggarwal B, Kumar A, Bharti A. Anticancer potential of curcumin: preclinical and clinical studies. Anticancer Res 2003;23(1A):363-98.
17. Sharma R, Gescher A, Steward W. Curcumin: the story so far. Eur J Cancer 2005;41:1955-68.

18. Shishodia S, Sethi G, Aggarwal B. Curcumin: getting back to the roots. Ann NY Acad Sci 2005;1056:206-17. 